\title{
ASTRONOMICAL DATA COORDINATION: A PERPETUAL TASK
}

\author{
W. D. Heintz, Vice-President, IAU Commission 5 \\ Department of Astronomy, Swarthmore College, \\ Swarthmore, PA 19081, USA
}

The conference now about to be concluded has presented a wide spectrum of coordinate efforts, of accomplishments, projects, and viewpoints. As is only natural and even purposeful in a first colloquium of this scope, there are also some loose ends which we hope to tie together later. Many experienced scientists found it worth participating in the subject which is more organization rather than primary research, and this is a very encouraging feature. Most of us will have deplored, in one context or other, the lack of reliable data compilations; yet not much could be done before efficient computer and storage facilities were at hand, and we may also have nourished the hope that someone else would undertake the tedious and often thankless job of compilation. We are grateful that the invitation to convene the first Numerical Data conference came from the Strasbourg Observatory, where a largescale and multi-purpose program has been successfully developing in recent years.

Bibliography has had a long history in astronomy and also in the IAU, along with the other organizational work in the commissions on ephemerides and on telegrams. Under the increasing wave of publications the tasks have greatly amplified and changed. How to channel the flood of results, whether digits or prose? Several new and viable working groups have formed: our consultant friends among the astronomical librarians, the group of journal editors, and here the specialists on numerical data. The groups represent astronomy in multi-disciplinary organizations like the ICSU Abstracting Board, the International Federation of Library Associations, and the CODATA committee, and they participate in work at the high level of UNESCO and ICSU. I believe that science-funding agencies in several countries are well aware of the increasing 
importance of secondary sources. Not only the primary data acquisition but also well-considered projects of compilation and retrieval should be found worth having some funds channelled into them.

More extended fields of science are still harder hit by the information explosion, as the decreased printing of full-length papers and the creation of synoptic journals show. Astronomy is also only marginally concerned with the intricate problems related to the commercial use of data. Yet we have problems of our own, for instance the long lifetime of many astronomical results, since many observations - unlike experiments - cannot be repeated at will, and a sizeable part of observing effort is for use in some future time. Results on a vast number of objects are to be kept separate since their value is individual as well as statistical, and moreover we have the peculiarly high fraction of publications in non-periodical, non-commercial forms with limited accessibility.

The enhanced responsibility carried by the author of a secondary source has been repeatedly mentioned, that is, with regard to the correctness of the data and - as the case may be - to their completeness or competent selection. Researchers are both producers and users of stored data, and the expedient and unambiguous presentation of data is needed for both ends of the supply line. Other items falling into the realm of the data trade are: to agree on standard formats for expedient processing, to find out what constitutes a feasible amount of redundancy and duplication, to advise on the extent or format in which data should continue to be published in traditional form, and perhaps also on the use of units and symbols. A special point may be made about the instructional value of the knowledge of sources, embodied in C. Jaschek's clear introduction to abstracts, catalogues, and data storage services this morning. Advanced students find it rewarding if at least one seminar period is devoted to the subject: How to use a library. An early ingrained know-how about records will save the prospective young astronomer countless hours of frustrating searches later on.

It was the work of the Strasbourg Observatory staff and their numerous helpers which rendered this conference a successful and pleasant event, in both the scientific and the social programs. V. Abalakine has already given a comprehensive tri-lingual catalogue at the Closing Dinner; so let me just join with our deeply felt thanks to our hosts. Let me also mention the indefatigable activity of our chairman, G. Wilkins. Compare what he has accomplished from the tentative beginnings in Sydney 1973 up to today.

Used though we are to work with printed catalogues, future data transfer surely will increasingly consist of communication with data centers. Science shall not be suffocated in the quick- 
sand of printed paper. Yet this meeting and particularly this morning's session will have made it abundantly clear that the preservation and utilization of what we already have is an indispensable foundation of high quality and efficiency of future research. 